\title{
Manejo de irrigação para cultivo de alface em ambiente protegido
}

\section{George RL Bandeira; Helder C dos S Pinto; Paula S Magalhães; Carlos A Aragão; Sérgio OP de Queiroz; Essione R Souza; Sirando L Seido}

UNEB-Dep ${ }^{\text {to }}$ Tecnologia e Ciências Sociais, Av. Edgard Chastinet, s/n, 48905-680 Juazeiro-BA; richbol_2@hotmail.com; heldercesarpinto@hotmail.com; paulasancho19@hotmail.com; carlosaragao@hotmail.com; sopqueiroz@gmail.com; essione.r@hotmail.com; siroseido@hotmail.com

\section{RESUMO}

A determinação do consumo de água de irrigação é fundamental para o manejo correto dos cultivos irrigados. Este trabalho teve o objetivo de estudar o desenvolvimento de duas cultivares da alface (AF 1743 e OGR 326), em ambiente protegido, no município de Juazeiro-BA, sob dois métodos de manejos de irrigação: reposição de água de irrigação determinada por tensiometria (M1) e reposição de água determinada na evaporação de água de tanque reduzido (M2), instalados em ambiente protegido. Observou-se efeito de interação entre os tratamentos impostos e as cultivares de alface estudadas, para a massa fresca das raízes das plantas. O uso da tensiometria promoveu desempenho superior para as variáveis de produção analisadas, como observado na massa fresca da parte aérea, apresentando média geral de 145,0 g, enquanto no manejo M2 tal valor foi de 59,4 g. Dentre as cultivares analisadas para referida variável, observou-se um resultado superior para a cultivar OGR 326 quando comparada à AF 1743.

Palavras-chave: Lactuca sativa, horticultura, manejo de água, evapotranspiração.

\section{ABSTRACT \\ Irrigation management for growing lettuce in greenhouse}

The determination of the water consumption is fundamental for the correct management of irrigated crops. The development of two lettuce cultivars (AF 1743 and OGR 326) was determined, when cultivated in a protected environment under two irrigation management methods: water consumption determined by the tensiometry (M1) and water consumption determined by evaporation of water in the reduced pan (M2), which were installed inside the protected environment. There was significant interaction among the imposed treatment and the cultivars of lettuce studied, for the fresh weight of the roots of the plants. The use of tensiometry promoted superior performance for the analyzed production variables as observed in the fresh weight of the aboveground part, with an overall average of $145.0 \mathrm{~g}$, whereas for the management M2 the overall average was $59.4 \mathrm{~g}$. For this variable, among the cultivars a superior result was obtained for the cultivar OGR 326 in comparison to AF1743.

Keywords: Lactuca sativa, horticulture, water management, evapotranspiration.

(Recebido para publicação em 23 de abril de 2010; aceito em 10 de maio de 2011) (Received on April 23, 2010; accepted on May 10, 2011)

A alface (Lactuca sativa L.) é uma hortaliça cultivada em todo o Brasil. No Sub Médio São Francisco, as condições edafoclimáticas favoráveis à irrigação, possibilitam o seu cultivo durante todo o ano, principalmente em hortas comunitárias. O sistema produtivo usualmente adotado, em campo aberto, não contempla a quantificação da lâmina de irrigação necessária e nem a exploração de cultivares superiores, caracterizadas pela alta produtividade e tolerâncias às viroses.

O cultivo de hortaliças em ambiente protegido é bastante difundido e aceito nas áreas de produção em todo o país. A sua aceitação e expansão entre produtores deve-se à exploração racional de pequenas áreas e à garantia de colheita, permitindo a obtenção de produções elevadas e de melhor qualidade (Queiroz et al., 2004).
Apesar da importância do cultivo em ambiente protegido para a olericultura brasileira, ainda são insuficientes os resultados de pesquisa que subsidiem o aproveitamento do potencial dessa tecnologia nas diferentes regiões climáticas do país, especialmente no que tange ao adequado manejo da irrigação (Santos \& Pereira, 2004). Este, por sua vez, permite o uso racional da água de irrigação e a obtenção de maiores produtividades com qualidade superior, especialmente para as cultivares de alta capacidade produtiva.

O manejo da irrigação, sob condições de ambiente protegido, pode basear-se em fatores do solo, do clima e da planta. O emprego isolado de sensores de solo, tanto para indicar o momento quanto para quantificar o volume de água a aplicar, surge como uma alternativa viável, com baixo custo e relativa praticidade (Figueiredo, 1998). Todavia, o monitoramento agroclimático no interior de ambientes protegidos enfrenta dificuldades em decorrência do espaço reduzido para a instalação de equipamentos. Segundo Fernandes et al. (2004), os produtores consideram inviável o comprometimento de uma área produtiva de aproximadamente 10 $\mathrm{m}^{2}$ para a instalação de equipamentos como o tanque Classe A.

Em ambiente protegido a evapotranspiração é em média menor que a verificada externamente, devido à redução da radiação solar e da ação dos ventos. Sob tais condições o déficit de vapor d'água é menor, afetando o crescimento e a produção indiretamente, através da influência na temperatura foliar, condutância estomatal e, especialmente, redução na área foliar, uma vez que a transpiração é também responsável pelo 
transporte de nutrientes para as folhas e outros órgãos, através da seiva do xilema (Cockshull, 1998).

Em métodos baseados na estimativa da evapotranspiração, as necessidades hídricas da cultura são expressas mediante a taxa de evapotranspiração (ET) em $\mathrm{mm} /$ dia ou $\mathrm{mm} /$ período. A ET está relacionada à demanda evaporativa do ambiente, que pode ser expressa pela evapotranspiração de referência (ETo), como forma de predição do efeito do clima sobre o consumo de água da cultura (Doorenbos \& Kassan, 2000).

Os tanques de evaporação têm sido estudados para quantificar indiretamente a demanda evaporativa do ambiente (Buriol et al., 2001; Vásquez et al., 2005; Koetz et al., 2006), nos quais a evapotranspiração é determinada multiplicando-se a evaporação do tanque por um coeficiente de correção (coeficiente do tanque, doravante denominado $\mathrm{Kp}$ ) a ser determinado para as condições locais, de acordo com Doorenbos \& Kassam (2000). Tem-se adotado tanques de evaporação com dimensões reduzidas como alternativa para estimativa da evapotranspiração devido à alta eficiência, menores dimensões, apresentando menor custo e à maior facilidade no manejo pelos agricultores (Menezes Junior et al., 1999).

Para a determinação do Kp, levam-se em consideração dados climáticos no interior do ambiente protegido, como umidade relativa e velocidade do vento, como referenciados na equação de regressão de Snyder (1992). Contudo, existem recomendações do uso do $\mathrm{Kp}$ com valor pre-determinado igual a 1,0, como descrito por Evangelista \& Pereira. (2003).

Esse trabalho teve o objetivo de avaliar o comportamento produtivo de duas cultivares de alface em ambiente protegido, sob dois métodos de manejo de água de irrigação, nas condições de clima semi-árido.

\section{MATERIAL E MÉTODOS}

O experimento foi conduzido em ambiente protegido no Departamento de Tecnologia e Ciências Sociais da Universidade do Estado da Bahia, município de Juazeiro (9'25'10'S; 40²9'15'W; $368 \mathrm{~m}$ de altitude), entre 25 de agosto e 03 de novembro de 2008.

O clima, segundo a classificação de Köeppen-Geiger, é do tipo semi-árido (BSwh), com valores médios anuais de precipitação de $542 \mathrm{~mm}$, temperatura anual de $28^{\circ} \mathrm{C}$ e umidade relativa do ar de $65 \%$.

O delineamento estatístico utilizado foi o inteiramente casualizado, em esquema de parcelas subdivididas, com dez repetições por tratamento, constituídos de dois manejos de irrigação $\left(M_{1}\right.$ e $\left.M_{2}\right)$ nas parcelas e duas cultivares de alface (AF 1743 e OGR 326) nas subparcelas. Os tratamentos referentes ao manejo de irrigação foram reposição de água de irrigação determinado pelo método de tensiometria ao atingir a tensão de $30 \mathrm{kPa}$ (M1); e reposição de água determinado com base em leituras de evaporação no tanque reduzido no interior da casa de vegetação adotando-se o Kp=1,0 (M2).

As mudas da alface foram produzidas em bandejas de poliestireno expandido, com 200 células, sendo utilizado um substrato comercial, ao qual foram adicionados $60 \mathrm{~g}$ do adubo fosfato de rocha de Irecê $\left(18 \% \mathrm{P}_{2} \mathrm{O}_{5}\right)$, sendo transplantadas para local definitivo após 38 dias da semeadura. Na adubação de plantio em local definitivo, foram utilizados $90 \mathrm{~kg} \mathrm{ha}^{-1}$ de uréia $(45 \%$ de $\mathrm{N})$, $555 \mathrm{~kg} \mathrm{ha}^{-1}$ do adubo fosfato de rocha de Irecê $\left(18 \%\right.$ de $\left.\mathrm{P}_{2} \mathrm{O}_{5}\right)$ e $133 \mathrm{~kg} \mathrm{ha}^{-1}$ de nitrato de potássio $\left(60 \%\right.$ de $\left.\mathrm{K}_{2} \mathrm{O}\right)$. Em cobertura, foram aplicados por vez, 44 $\mathrm{kg} \mathrm{ha}^{-1}$ de uréia ( $45 \%$ de N), aos 10 e 20 dias após o transplantio.

As mudas foram acondicionadas em vasos plásticos, com dimensões de $20 \mathrm{~cm}$ de altura, diâmetro de $21 \mathrm{~cm} \mathrm{e}$ volume de 4,5 L. O substrato utilizado foi composto por uma mistura de dois solos, adicionada ao esterco de caprino na proporção de $3: 1$, resultando numa densidade total de $1,43 \mathrm{~g} \mathrm{~cm}^{-3}$ e caracterizado como de textura média.

A análise química do substrato para a condução do experimento apresentou: $\mathrm{pH}\left(\mathrm{H}_{2} \mathrm{O}\right)=7,3 ; \mathrm{K}^{+}=0,56 \mathrm{cmol} \mathrm{dm}^{-3}$; $\mathrm{P}=45,0 \mathrm{mg} \mathrm{dm}^{-3} ; \mathrm{Ca}=9,0 \mathrm{cmol} \mathrm{dm}^{-3}$; $\mathrm{Mg}=2,8 \mathrm{cmol} \mathrm{dm}^{-3} ; \mathrm{Na}=0,13 \mathrm{cmol}$ $\mathrm{dm}^{-3} ; \mathrm{Al}=0,0 \% ;$ M.O. $=1,05 ; \mathrm{T}=12,66$; $\mathrm{V} \%=98,64$.
Para o manejo M1, determinou-se o teor de água no solo sob tensões variadas, utilizando-se um recipiente plástico de dez litros e uma bateria de três tensiômetros inseridos no substrato acondicionado no mesmo. Inicialmente foi feita a saturação do solo por ascensão capilar, imergindo o recipiente plástico em bandeja contendo água, em valores próximos ao equilíbrio dinâmico entre potencial gravitacional e matricial. $\mathrm{O}$ mesmo foi pesado, quando então se iniciou a mensuração das tensões e teor volumétrico de água, através do uso de um tensiômetro digital de punção e balança com precisão de 0,01 g.

Esses dados coletados permitiram a elaboração da curva de retenção, confeccionada a partir de uma equação logarítmica (Figura 1), com valores iniciais de tensão de $10 \mathrm{KPa}$ (capacidade de campo-Cc) até a tensão de $40 \mathrm{KPa}$. Para o manejo por tensiometria, após o transplante de mudas, foram instalados quatro tensiômetros em vasos escolhidos aleatoriamente, depois da saturação em ambiente protegido. A tensão inicial adotada foi de $10 \mathrm{kPa}$, correspondente à $\mathrm{Cc}$ e a de reposição, de $30 \mathrm{kPa}$ para indicar o momento de irrigar. $\mathrm{O}$ preparo e instalação dos tensiômetros foram feitos de acordo com as recomendações de Marouelli (2008), mantidas as mesmas condições de densidade e composição do substrato, além da profundidade de inserção dos tensiometros, viabilizando o uso da curva característica obtida na fase anterior.

O minitanque utilizado foi confeccionado em PVC, apresentando dimensões de $50 \mathrm{~cm}$ de diâmetro e 39 $\mathrm{cm}$ de profundidade. A pintura interna e externa foi feita com tinta látex prata, e o tanque assentado em estrado de madeira com dimensões de $0,8 \times 0,8 \mathrm{~m}$ a $13 \mathrm{~cm}$ acima da superfície do solo. Sua colocação se deu na porção mediana do ambiente protegido, uma casa de vegetação construída no sentido leste-oeste, em estrutura de madeira e dimensões de 10,2 x 7,2 m e pé-direito de 2,13 m, coberta com filme de polietileno preto com $75 \%$ de sombreamento, de acordo com procedimento utilizado por Paula et al. (2005), para a instalação do tanque Classe A.

No manejo M2, os dados de evapora- 
ção foram coletados em leituras diárias às 7:00 $\mathrm{h}$ da manhã, com um parafuso micrométrico instalado no mini tanque.

O coeficiente adotado para o mini tanque, doravante denominado simplesmente como $\mathrm{kp}$, igual a 1,0 (um), foi o recomendado por Lopes Filho (2002), citado por Evangelista \& Pereira (2003).

A quantidade da água de irrigação para aplicação em $\mathrm{mL}$, no tratamento M2 foi determinada a partir do conhecimento da evapotranspiração real (ETR em mm), obtida de acordo com a fase fenológica da cultura. Para a aplicação utilizou-se uma proveta de $500 \mathrm{~mL}$, calculando-se o volume a ser aplicado de acordo com a área do vaso e a ETR: $\mathrm{Vol}=1000 \times \mathrm{Av} \times \mathrm{ETR}$, em que $\mathrm{Vol}=$ Volume de água a ser aplicado, em $\mathrm{mL}$; $\mathrm{ETR}=$ evapotranspiração real, em mm (1 $\left.\mathrm{mm}=1 \mathrm{~L} / \mathrm{m}^{2}\right) ; \mathrm{Av}=$ Área da superfície do vaso, em $\mathrm{m}^{2}$.

O manejo cultural da alface foi feito de acordo com as recomendações de plantio para a região, sendo a colheita das plantas realizada trinta dias após o transplante, avaliando-se as características: massa fresca da parte aérea (MFPA), massa seca da parte aérea (MSPA), massa fresca de raízes (MFR), massa seca de raízes (MSR) e diâmetro de rosetas (DR), representadas pela média de dez plantas. A eficiência do uso da água foi determinada pela relação entre a produtividade da massa fresca da parte aérea (MFPA) e quantidade de água aplicada através da irrigação, como descrito por Doorenbos \& Kassam (2000).

A análise estatística dos resultados foi realizada por meio da análise de variância dos dados (teste de F) e da comparação de médias de tratamentos entre si (teste de Tukey, $5 \%$ de probabilidade).

\section{RESULTADOS E DISCUSSÃO}

Os dados de evapotranspiração de referência (ETo) estimados a partir dos valores de evaporação do minitanque no período de condução do experimento totalizaram 130,14 $\mathrm{mm}$ no manejo de irrigação M2, correspondendo a 64,42\% daqueles encontrados a partir dos dados de evaporação do tanque Classe A em ambiente externo (202,0 mm). Estes resultados demonstram que os valores da

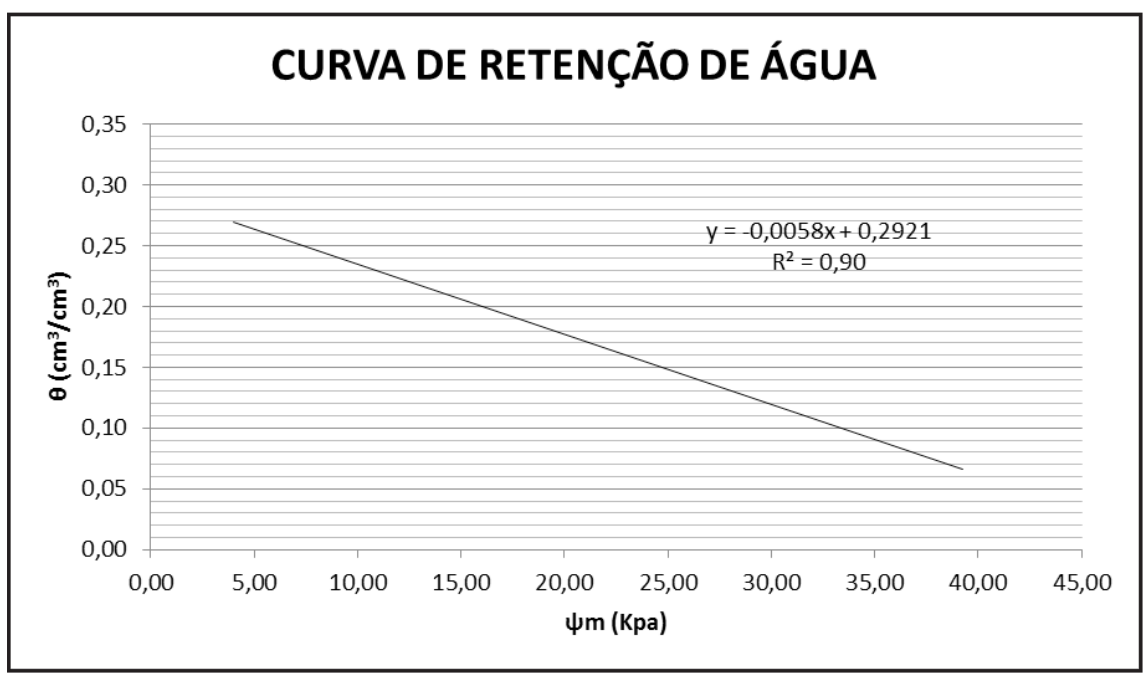

Figura 1. Curva de retenção de água (water retention curve). Juazeiro, UNEB, 2008.

evapotranspiração de referência foram maiores em campo aberto, corroborando com informações obtidas por Fernandes et al. (2004). Isto se deve ao fato de a velocidade do vento e a radiação solar incidente serem maiores fora da casa de vegetação do que no interior da mesma (Martins et al., 1994), podendo levar a um consumo hídrico das espécies cultivadas em ambiente protegido entre 20 e $40 \%$ inferior àquele observado ao cultivo em céu aberto.

Para o manejo M1, baseado na tensiometria, a lâmina total de irrigação correspondeu a $280 \mathrm{~mm}$ enquanto que para o manejo M2 esse valor foi de $130,14 \mathrm{~mm}$ indicando um consumo de $46,48 \%$ desse manejo, quando comparado ao M1. Estes resultados demonstram que o uso do minitanque, para as condições experimentais, subestimou a necessidade de reposição de água das plantas de alface, induzindo o déficit hídrico. Para Doorenbos \& Kassam (2000), a predição da evapotranspiração máxima (ETm) a partir da evapotranspiração de referência (ETo), através dos tanques de evaporação, podem resultar em erro que varia de 10 a $20 \%$, afastando a evapotranspiração real (ETr) da evapotranspiração máxima. Tal subestimativa pode dever-se à área exposta e profundidade do tanque, associadas à inexistência de coeficientes de cultivo específicos para a região e para as cultivares avaliadas. Para a região do Vale do São Francisco não existem referências de uso de tanques reduzidos para estimativa da evapotranspiração de cultivo em ambiente protegido; assim, optou-se por tanque de dimensões superiores àquelas utilizadas em outras regiões do país, como forma de aproximar as leituras obtidas daquelas do tanque Classe A.

A exposição de plantas ao estresse hídrico, por sua vez, induz ao declínio do potencial de água na folha, da condutância estomática e do fluxo de $\mathrm{CO}_{2}$, podendo representar um impacto adverso sobre o acúmulo de fotoassimilados e produtividade, como observado por diversos autores em beterraba açucareira (Tognetti et al., 2003), feijão (Paiva et al., 2005; Selen et al., 2008) e milho (Payero et al., 2008). Ainda sob tais condições, tem-se observado elevação na concentração de ácido abscísico (ABA) no xilema em feijão (Oliveira $e t$ $a l ., 2002)$ e laranja pêra (Gomes et al., 2004). Sob estresse hídrico, diferentes espécies podem apresentar mecanismos de sobrevivência que podem incluir variações nas respostas estomáticas, ajuste osmótico e maior movimento de fotoassimilados para as raízes (Jaimez et al., 1999), com consumo de energia metabólica, podendo afetar a produção de plantas e sua qualidade comercial.

Por outro lado, a eficiência de uso de água (Ea) indica valores de 136,29 kg/ $\mathrm{ha} / \mathrm{mm}$ para o manejo M1 e $120,11 \mathrm{~kg} /$ ha/mm para o manejo M2. Andrade Júnior (1992) obteve uma função linear decrescente entre níveis de água aplicados com base em tanque Classe A e Ea em cultivar de alface americana, mostrando que os tratamentos com maior lâmina aplicada tenderam a apresentar menor 
Tabela 1. Resultados médios de massa fresca de raízes (MFR); massa fresca da parte aérea (MFPA); massa seca da parte aérea (MSPA); massa seca de raízes (MSR) e diâmetro de roseta (DR) de cultivares de alface, submetidas a diferentes manejos de água (average results of fresh weigh of roots (MFR); fresh weight of aboveground part (MFPA); dry weight of aboveground part (MSPA); dry weight of roots (MSR) and head diameter (DR) of lettuce cultivars, submitted to different water regimes). Juazeiro, UNEB, 2008.

\begin{tabular}{lcccccc}
\hline \multirow{2}{*}{ Manejos de água } & \multicolumn{2}{c}{ MFR (g) } & \multirow{2}{*}{ MFPA (g) } & \multirow{2}{*}{ MSPA (g) } & \multirow{2}{*}{ MSR (g) } & \multirow{2}{*}{ DR (cm) } \\
\cline { 2 - 3 } & AF 1743 & OGR 326 & & & \\
M1 Tensiometria & $71,40 \mathrm{aA}$ & $30,10 \mathrm{aB}$ & $145,00 \mathrm{a}$ & $10,66 \mathrm{a}$ & $2,35 \mathrm{a}$ & $36,42 \mathrm{a}$ \\
M2 Eto com Kp =1 & $49,30 \mathrm{bA}$ & $26,60 \mathrm{aB}$ & $59,40 \mathrm{~b}$ & $7,32 \mathrm{~b}$ & $1,80 \mathrm{a}$ & $29,99 \mathrm{~b}$ \\
\hline CV (\%) & & 15,61 & 16,30 & 77,75 & 8,3 \\
\hline Cultivares & & & & \\
\hline AF 1743 & $97,00 \mathrm{~b}$ & $10,00 \mathrm{a}$ & $3,47 \mathrm{a}$ & $34,09 \mathrm{a}$ \\
OGR 326 & & $107,40 \mathrm{a}$ & $7,97 \mathrm{~b}$ & $0,66 \mathrm{~b}$ & $32,32 \mathrm{~b}$ \\
\hline CV (\%) & & 14,02 & 17,78 & 72,47 & 7,27 \\
\hline
\end{tabular}

Média seguidas de mesma letras minúsculas nas colunas e maísculas nas linhas, não diferem entre si pelo teste de Tukey a 5\% de probabilidade (means followed by same capital letters in lines and lower case letters in columns, are not different by the Tukey test, $\mathrm{p}<0.05$ ).

Ea, resultado não observado nesse experimento para as cultivares estudadas.

Houve efeito de interação entre os diferentes manejos de água utilizados e as cultivares de alfaces estudadas, para a massa fresca das raízes (Tabela 1). A reposição de água pelo manejo $\mathrm{M} 1$ (tensiometria) promoveu uma maior produção de biomassa de raízes, com médias de 71,4 e 30,1 gramas para as cultivares AF 1743 e OGR 326, respectivamente (Tabela 1). Silva et al. (1998), afirmam que hortaliças cultivadas em ambiente protegido e manejadas por gotejamento, de modo geral, apresentam melhor desempenho quando submetidas a tensões da água no solo mais próximas à capacidade de campo, isto é, entre 10 e $30 \mathrm{kPa}$. Através da análise isolada das cultivares dentro de cada manejo de água, constatou-se comportamento superior da cv. AF 1743, significativamente a $5 \%$ de probabilidade, quando comparada com a cv. OGR 326, nos dois métodos de irrigação para a massa fresca das raízes (Tabela 1).

Santos \& Pereira (2004), estudando diferentes tensões de água no solo, observaram que há fortes evidências de haver uma redução na altura das plantas em intervalos estudados de 15 a $89 \mathrm{kPa}$, sugerindo que quanto mais próximo à tensão correspondente à capacidade de campo, mais altas são as plantas de alface. No mesmo trabalho, a matéria fresca da parte comercial (MFPC) das plantas apresentou resposta linearmente inversa ao aumento da tensão, com a obtenção dos melhores resultados na tensão de 15
$\mathrm{kPa}$. Já com relação ao teor de matéria seca da parte comercial (TMSPC), este aumentou linearmente com o aumento da tensão.

Para o manejo M2, a cv. AF 1743 apresentou também melhor performance em relação a OGR 326, diferindo desta ao produzir em média $49,3 \mathrm{~g}$ de massa fresca de raízes, contra $26,6 \mathrm{~g}$ produzida pela segunda cultivar (Tabela 1).

Para a massa fresca da parte aérea, não houve efeito de interação entre os dois fatores estudados. Isoladamente o manejo M1, proporcionou um maior incremento de massa fresca da parte aérea para as cultivares de alface, com média geral de $145,0 \mathrm{~g}$, enquanto que o manejo M2 apresentou valor 59,4 g (Tabela 1). Com relação às cultivares, a cv. OGR 326 apresentou maior massa fresca da parte aérea, com 107,4 g, sendo tal atributo o mais importante a ser considerado na qualidade do produto final.

Andrade Júnior et al. (1992), analisando os efeitos de quatro níveis de irrigação baseados na evaporação do Tanque Classe A (ECA) $(0,5 ; 0,75 ; 1,0$ e 1,25$)$ aplicados por microaspersão em alface, constataram que a matéria fresca da "cabeça" e a produtividade apresentaram resposta quadrática, tendo alcançado os valores máximos de 184 g e 23,67 tha ${ }^{-1}$, respectivamente, com o nível de irrigação correspondente a $75 \%$ da ECA. Os resultados relativos à eficiência do uso de água revelaram resposta linear decrescente com o aumento dos níveis de irrigação, significando que a cultura responde melhor à aplicação de pequenas lâminas de irrigação, porém com alta frequência.

Para a variável massa seca da parte aérea, os efeitos dos fatores, cultivares e manejos de água, foram independentes. O manejo M1, foi o que proporcionou maior massa seca de parte aérea, com média de 10,66 g, enquanto o manejo M2 apresentou plantas com 7,32 g de massa seca da parte aérea (Tabela 1).

Quando se analisou o diâmetro de rosetas, novamente a cultivar AF 1743 , suplantou a OGR 326, com formação de rosetas com $34,09 \mathrm{~cm}$ e $32,32 \mathrm{~cm}$ de diâmetro médio respectivamente (Tabela 1). Quanto aos manejos de água avaliados, relacionados ao diâmetro formado, percebe-se que o manejo M1 induziu a formação de rosetas maiores.

Hamada (1993), objetivando analisar a resposta da alface à aplicação de diferentes lâminas de irrigação baseadas na evaporação de um Tanque Classe A (ECA) $(0,6 ; 0,8 ; 1,0$ e 1,2) utilizando irrigação por gotejamento, verificou que os melhores resultados de produtividade e produção de plantas de melhor qualidade comercial foram obtidos com a aplicação do nível de irrigação equivalente a 1,2 vezes a evaporação do Tanque Classe A. Entretanto, a maior eficiência do uso de água foi obtida no tratamento com a menor lâmina de irrigação aplicada (0,6 da ECA).

Pode-se concluir que, nas condições experimentais avaliadas, o manejo de água por tensiometria promoveu melhor desempenho para as variáveis analisadas 
e maior eficiência no uso da água. A cultivar OGR 326, apresentou comportamento superior para a produção de massa fresca de parte aérea.

\section{REFERÊNCIAS}

ANDRADE JUNIOR AS; DUARTE RLR; RIBEIRO VQ. 1992. Niveis de irrigação na cultura da alface. Teresina: EMBRAPAUEPAE. 16p. (Boletim de Pesquisa, 13).

BURIOL GA; LUZZA J; HELDWEIN AB; STRCK NA. 2001. Evaporação d'água em estufas plásticas e sua relação com o ambiente externo: 1 - avaliação com uso de tanque classe A e do evaporímetro de Piche. Revista Brasileira de Agrometeorologia 9: 35-41.

COCKSHULL KE. 1998. Plant responses and adaptation to water issues in the greenhouse environment. Acta Horticulturae 379: 187192.

DOORENBOS J; KASSAM AH. 2000. Efeito da água no rendimento das culturas. Campina Grande: Tradução Gheyi H.R. e outros, UFPb FAO. 221p.

EVANGELISTA AWP; PEREIRA GM. 2003. Avaliação de dois tipos de evaporímetros na estimativa da demanda evaporativa do ar (ET) no interior de casa de vegetação, em LavrasMG. Ciência e Agrotecnologia 27: 1348-1353.

FERNANDES C; CORÁ JE; ARAÚJO AC. 2004. Utilização do tanque Classe A para a estimativa da evapotranspiração de referência dentro da casa de vegetação. Engenharia Agrícola 24: 46-50.

FIGUEIRÊDO SF. 1998. Estabelecimento do momento de irrigação com base na tensão de água no solo para a cultura do feijoeiro. Piracicaba: USP-ESALQ. 72p. (Tese mestrado).

GOMES MMA; LAGOAAMMA; MEDINA CL; MACHADO EC; MACHADO MA. 2004. Interactions between leaf water potential, stomatal conductance and absisic acid content of orange trees submitted to drought stress. Brazilian Journal Plant Physiology. 16: 155-161.

HAMADA E. 1993. Desenvolvimento e produtividade da alface (Lactuca sativa L.), submetida a diferentes lâminas de irrigação, através da irrigação por gotejamento. Campinas: UNICAMP-FAEA. 102p. (Tese mestrado).

JAIMEZ RE; RADA F; GARCIA-NUÑEZ C. 1999. The effect of irrigation frequency on water and carbon relations in three cultivars of sweet pepper (Capsicum chinense Jacq), in a tropical semiarid region. Scientia Horticulturae 81: 301-305.

KOETZ M; COELHO G; CARVALHO JA; SOUZA RJ; SILVA RA. 2006. Produção do meloeiro em ambiente protegido irrigado com diferentes lâminas de água. Irriga 11: 500-506.

MARTINS G; CASTELLANE PD; VOLPE CA. 1994. Influência da casa de vegetação nos aspectos climáticos e em época de verão chuvoso. Horticultura Brasileira 12: 131-135.

MAROUELLI WA. 2008. Tensiômetros para o controle da irrigação em hortaliças. Brasília: EMBRAPA/CNPH.15p. Circular Técnica, 57.

MENEZES JUNIOR FOG; MARTINS SR; DUARTE GB; FORTES DF 1999. Estimativa de evapotranspiração em ambiente protegido mediante a utilização de diferentes evaporímetros. In: CONGRESSO BRASILEIRO DE AGROMETEOROLOGIA, 11. Anais... Florianópolis: SBA. p. 2130-2136.

OLIVEIRA MNS; OLIVA MA; MARTÍNEZ CA; MERCIER H; SILVA MAP. 2002. Sensibilidade estomática ao ABA em função do $\mathrm{pH}$ e de níveis de $\mathrm{Ca}^{2+}, \mathrm{NO}_{3}{ }_{3}^{-} \mathrm{PO}_{4}{ }^{3-}$ na seiva do xilema. Brazilian Journal Plant Physiology. 14: 117-123.

PAIVA AS; FERNANDES EJ; RODRIGUES TJD; TURCO JEP. 2005. Condutância estomática em folhas de feijoeiro submetido a diferentes regimes de irrigação. Engenharia Agrícola 25: 161-169.

PAULA VA; ANDRADE FF; DUARTE GRB; ÁVILA MSV; CASTRO RC, DUARTE L;
FRAGA DS; MENDEZ MEG; SCHÖFFEL ER. 2005. Variação espacial da evaporação de tanques reduzidos em ambiente protegido cultivado com tomateiro. Pelotas: UFPel. $4 \mathrm{p}$.

PAYERO JO; TARKALSON DD; IRMAK S; DAVISON D; PETERSEN JR. 2008. Effect of irrigation amounts applied with subsurface drip irrigation on corn evapotranspiration, yield, water use efficiency, and dry matter production in semiarid climate. Agricultural Water Management 95: 895-908.

QUEIROZ SOP; TESTEZLAF R; MATSURA EE. 2004. Avaliação de equipamentos para a determinação da condutividade elétrica do solo. Irriga 10: 279-287.

SANTOS SR; PEREIRA GM. 2004. Comportamento da alface americana sob diferentes tensões da água no solo, em ambiente protegido. Engenharia Agrícola 24: $569-577$.

SELEN SM; AKYILDIZ A; DASGAN HY; GENCEL B. 2008. Yield and quality response of drip irrigated green beans under full and deficit irrigation. Scientia Horticulturae 117: 95-102.

SILVA WLC; MAROUELLI WA. 1998. Manejo da irrigação em hortaliças no campo e em ambientes protegidos. In: FARIA MA; SILVA EL; VILELA LAA; SILVA AM (eds). Manejo de irrigação. Poços de Caldas: UFLA/SBEA, p.311-348.

SNYDER RL. 1992. Equation for evaporation pan to evapotranspiration conversions. Journal of Irrigation and Drainage Engineering 118: 977-980.

TOGNETTI R; PALADINO M; MINNOCCI A; DELFINE S; ALVINO A. 2003. The response of sugar beet to drip and low-pressure sprinkler irrigation in southern Italy. Agricultural Water Management 60: 135-155.

VASQUÉZ MAN; FOLEGATTI MV; DIAS NS; SILVACR. 2005. Efeito do ambiente protegido cultivado com melão sobre os elementos meteorológicos e sua relação com as condições externas. Engenharia Agrícola 25:137-143 